\title{
Early single-center experience in sutureless aortic valve implantation in 120 patients
}

\author{
Harald C. Eichstaedt, MD, ${ }^{a}$ Jerry Easo, MD, ${ }^{a}$ Tobias Härle, MD, ${ }^{b}$ and Otto E. Dapunt, MD, PhD ${ }^{\mathrm{a}}$
}

Objective: The study objective was to evaluate the safety and efficacy of sutureless self-expanding nitinol stentframe aortic valve prostheses made of equine pericardium implanted in patients with symptomatic aortic valve disease.

\begin{abstract}
Methods: We performed a retrospective analysis of 120 patients (mean age, $76.7 \pm 5.9$ years) who underwent isolated aortic valve replacement or in combination with other cardiovascular procedures. Preoperatively, Society of Thoracic Surgeons score was determined. Transthoracic echocardiography and clinical outcome evaluation were performed at the time of discharge and at 6,12 , and 18 months follow-up, respectively.
\end{abstract}

Results: A total of 71 of 120 patients underwent isolated sutureless aortic valve replacement (mean aortic crossclamp time, $37 \pm 11$ minutes; mean bypass time, $62 \pm 18$ minutes). Coronary bypass grafting was performed in 30 patients. Overall mean Society of Thoracic Surgeons score was $14.8 \% \pm 10 \%$. Thirty-day mortality rate was $6.7 \%$ overall and $1.4 \%$ in stand-alone procedures. During a mean follow-up of 313 days, 3 more deaths occurred. The reoperation rate was $4.2 \%$. Mean and peak transvalvular pressure gradients were $9 \mathrm{~mm} \mathrm{Hg}(4-13$ $\mathrm{mm} \mathrm{Hg})$ and $14 \mathrm{~mm} \mathrm{Hg}(8-22 \mathrm{~mm} \mathrm{Hg})$ at discharge, respectively. In 8 patients $(6.7 \%)$, permanent pacemaker implantation was necessary. No thromboembolic events or bleedings related to the bioprosthesis were observed.

Conclusions: In this large single-center experience with sutureless aortic valve replacement, the surgical procedure is shown to be safe and time-saving. In view of the excellent hemodynamic results and shortening of aortic crossclamp and bypass times, we notice advantages especially in high-risk patients. Minimally invasive access seems to be facilitated. The long-term durability of this prosthesis has yet to be determined. (J Thorac Cardiovasc Surg 2014;147:370-5)

Despite the recent introduction of various alternative approaches, surgical treatment of the aortic valve represents the gold standard of treatment in case of severe and symptomatic valve disease. This is also proven for various patients with multiple comorbidities and high perioperative risk, for example, older patients and patients with a distinct prosthesis-patient mismatch. ${ }^{1-3}$ Various surgical approaches to enhanced treatment of the diseased aortic valve have been developed in the last decade, ranging from aortic valve repair to stentless prosthesis and complex aortic root surgery. ${ }^{4-6}$

Numerous studies have shown pressure gradients across the implanted prosthesis to be crucial for mass regression and restoration of left ventricular function and, consequently, long-term survival. ${ }^{7,8}$ Stentless substitutes have been shown to be surgically more challenging but result in superior hemodynamics because of a larger effective

\footnotetext{
From the Departments of Cardiovascular Surgery ${ }^{\mathrm{a}}$ and Cardiology, ${ }^{\mathrm{b}}$ Klinikum Oldenburg, Oldenburg, Germany.

Disclosures: Drs Eichstaedt and Dapunt serve as proctors for Medtronic, Inc (Minneapolis, Minn). The other authors have nothing to disclose with regard to commercial support.

Received for publication July 12, 2012; revisions received Dec 7, 2012; accepted for publication Dec 12, 2012; available ahead of print March 7, 2013.

Address for reprints: Otto E. Dapunt, MD, PhD, Department of Cardiovascular Surgery, Klinikum Oldenburg, Rahel-Straus-Str 10, 26133 Oldenburg, Germany (E-mail: dapunt.otto@klinikum-oldenburg.de). $0022-5223 / \$ 36.00$

Copyright $\Subset 2014$ Published by Elsevier Inc. on behalf of The American Association for Thoracic Surgery

http://dx.doi.org/10.1016/j.jtcvs.2012.12.062
}

orifice area and omission of the obstructive elements of the stented bioprosthesis. In this context, the replacement of the aortic valve with a sutureless prosthesis held in place by radial forces represents an interesting idea. The symmetric pericardial prosthesis is mounted onto a nitinol frame, which guarantees optimal orientation because of its memory effect. ${ }^{9}$ This new technique offers complete removal of the diseased aortic valve and calcifications under vision in combination with avoiding placement and tying of sutures in the aortic annulus, facilitating minimally invasive access, decreasing surgical trauma, and providing hemodynamic benefits through its unique design.

Various reports have been published on the initial experience in relatively small cohorts of selected patients, mostly with isolated aortic valve stenosis. ${ }^{10-12}$ The current study reports on the largest single-center experience in sutureless implantation of the Enable aortic bioprosthesis (Medtronic, Inc, Minneapolis, Minn) in unselected patients with respect to their clinical outcome and hemodynamic performance for up to 18 months postoperatively.

\section{MATERIALS AND METHODS \\ Patients}

Between July 2010 and March 2012, 120 patients were included in this study. Inclusion criteria were severe, symptomatic aortic valve disease, New York Heart Association function class II or higher, and scheduled for surgical valve replacement. All patients gave written informed consent 


\section{Abbreviations and Acronyms \\ AVR $=$ aortic valve replacement \\ TEE $=$ transesophageal echocardiography}

except in case of emergency. Detailed patient characteristics are listed in Table 1.

\section{Sutureless Aortic Valve Prosthesis}

The Enable sutureless aortic prosthesis consists of 2 main parts: the original stentless $3 \mathrm{~F}$ biological valve made of equine pericardium with 3 symmetric sinuses that are incorporated into a nitinol stent frame. This material returns to its original shape when it is exposed to body temperature while maintaining the correct orientation of the pericardial leaflets.

\section{Operative Technique}

Cardiopulmonary bypass and cardioplegic arrest are established in routine fashion after median or partial sternotomy. In selected patients with a minimally invasive approach, a small right anterolateral minithoracotomy approximately $7 \mathrm{~cm}$ in length is performed and cannulation for cardiopulmonary bypass is installed via femoral cannulation. Because of the height of the nitinol stent of the prosthesis, a transverse aortotomy at least $3.5 \mathrm{~cm}$ above the origin of the ostium of the right coronary artery is mandatory. The diseased native aortic leaflets are excised carefully without injuring the annulus. Precise sizing of the aortic annulus is crucial. The prosthesis chosen is then rinsed for $30 \mathrm{sec}-$ onds 3 times before placing it into ice-cold water. When malleable, the prosthesis is wrapped around a plastic bar and mounted onto a cross-action forceps.

A double-armed 4-0 polypropylene suture is placed into the nadir of the noncoronary sinus and the corresponding site at the upper flange of the Enable ring. The folded Enable prosthesis is then positioned into the aortic annulus supported by the guiding suture. After removal of the forceps, the stent is unfolded and aligned to the remaining aortic annulus. Meticulous and precise placement of the valve prosthesis along the aortic annulus is absolutely mandatory for successful deployment. Correct positioning is verified followed by application of warm water causing the nitinol stent to regain its original shape.

After weaning from cardiopulmonary bypass with cannulas still in place, transesophageal echocardiography (TEE) is performed to evaluate correct positioning of the prosthesis and to exclude paravalvular leakage. In patients with concomitant cardiac procedures, placement of the Enable prosthesis is performed as the last procedure.

\section{Follow-up}

All data related to the clinical outcome of the patient and the performance of the Enable prosthesis were collected. Thereby, adverse events were divided into early (within 30 days postoperatively) and late complications (after 30 days).

Before discharge, every patient underwent investigation of the Enable prosthesis by 2-dimensional transthoracic echocardiography. Hemodynamic parameters, such as transvalvular peak and mean pressure gradients, and effective orifice area were determined; these echocardiographic examinations were repeated 6,12 , and 18 months after surgery, respectively.

\section{Statistical Analysis}

For general demographic, clinical, and operative data, descriptive statistical methods were used and expressed as mean \pm standard deviation. Categoric variables are presented as numbers with percentages.

\section{RESULTS}

Of the 120 patients, the majority was male (81/120; $67.5 \%$ ). The mean age at the time of implantation was
$76.7 \pm 5.9$ years (range, $64-90$ years). More than $90 \%$ of the patients were in New York Heart Association class III and IV. The foremost cause for valve replacement was severe stenosis due to degenerative or rheumatic valve disease $(110 / 120 ; 91.7 \%)$. Ten patients presented with severe pure aortic insufficiency as the underlying valve pathology. In 3 of these patients, valve replacement was necessary because of acute and subacute endocarditis. Detailed patient demographics are depicted in Table 1.

The overall study patients' mean logistic European System for Cardiac Operative Risk Evaluation was 20.71\% (range, 2\%-90\%), and the mean Society of Thoracic Surgeons score was $14.78 \%$ (range, $2 \%-76 \%$ ) for overall predictive mortality. However, for patients with isolated aortic valve replacement (AVR) the predictive risk scores were slightly lower (16.3\% and $12.1 \%$, respectively) (Table 2 ).

Isolated implantation of the Enable prosthesis in the aortic position was performed in 71 patients $(59.2 \%)$. Although two thirds of those underwent a full sternotomy, a less-invasive approach was chosen in the remaining patients undergoing isolated AVR: partial sternotomy $(n=20)$ or anterolateral minithoracotomy $(n=4)$ in selected patients.

A total of 49 patients underwent concomitant surgical procedures, with coronary bypass surgery being the most frequent $(30 / 49 ; 61.2 \%)$. The number of bypass grafts ranged from 1 to 4 arterial and venous grafts per patient. The remaining patients in the concomitant group (19/49 patients; $38,8 \%$ ) underwent various concurrent surgeries, listed in Table 3.

In the isolated AVR group, mean cardiopulmonary bypass time was $62 \pm 18$ minutes (range, 39-141 minutes) and mean crossclamp time was $37 \pm 11$ minutes (range, 18-69 minutes). In the group of patients who underwent concomitant procedures, crossclamp and bypass times were mainly dependent on the extent of the additional procedures and were on average $47 \pm 19$ minutes (range, 18134 minutes) and $80 \pm 39$ minutes (range, 39-322 minutes), respectively.

All available valve sizes were implanted in patients in this study, with the majority of patients receiving a 23- or 25-mm valve. The valve size distribution was as follows: $19 \mathrm{~mm}(\mathrm{n}=2), 21 \mathrm{~mm}(\mathrm{n}=18), 23 \mathrm{~mm}(\mathrm{n}=48), 25$ $\mathrm{mm}(\mathrm{n}=32), 27 \mathrm{~mm}(\mathrm{n}=18)$, and $29 \mathrm{~mm}(\mathrm{n}=2)$.

A repeat aortic crossclamping was necessary intraoperatively in 10 patients because of paravalvular leakage $(n=7)$ and acute migration into the left ventricle $(\mathrm{n}=2)$ or into the ascending aorta $(\mathrm{n}=1)$ assessed by TEE while cannulas for cardiopulmonary bypass were still in place (Table 4). In patients with intraoperative paravalvular leakage $(n=7)$, the reasons for repeat crossclamping of the aorta were incorrect size in 3 patients and irregular annulus (ie, significantly different size and level of the 3 sinuses) in 4 patients, leading to improper positioning. A different size of the same type of 
TABLE 1. Preoperative patient characteristics

\begin{tabular}{lc}
\hline No. of patients & $120($ male, $67.5 \%)$ \\
Mean age & 77 y $(64-90 \mathrm{y})$ \\
Logistic euroSCORE (mean) & $20.7 \% \pm 19 \%$ \\
STS score (mean) & $14.8 \% \pm 10 \%$ \\
BMI & $27.8 \% \pm 4.7 \%$ \\
Ejection fraction & $55 \% \pm 11 \%$ \\
NYHA class & \\
II & $11 / 120(9.2 \%)$ \\
III & $93 / 120(77.5 \%)$ \\
IV & $16 / 120(13.3 \%)$ \\
Risk factors & \\
Pulmonary hypertension & $54 / 120(45 \%)$ \\
Carotid artery disease & $34 / 120(28.3 \%)$ \\
Stroke & $12 / 120(10 \%)$ \\
Atrial fibrillation & $28 / 120(23.3 \%)$ \\
Endocarditis & $3 / 120(2.5 \%)$ \\
Diabetes (under medication) & $27 / 120(22.5 \%)$ \\
Dialysis & $3 / 120(2.5 \%)$ \\
Chronic lung disease & $16 / 120(13.3 \%)$ \\
Dyslipidemia & $81 / 120(67.5 \%)$ \\
\hline Pulmonary hypertension = pulmonary artery pressure greater than $25 \mathrm{~mm} \mathrm{Hg}$ or in- \\
direct echocardiographic findings, such as dilatation or impairment of the right ven- \\
tricle function. Carotid artery disease = stenosis of internal carotid artery of $50 \%$ or \\
greater. Chronic lung disease = patients under long-term medication for chronic \\
bronchitis or emphysema and pathologic pulmonary function test. euroSCORE, \\
European System for Cardiac Operative Risk Evaluation; STS, Society of Thoracic \\
Surgeons; BMI, body mass index; NYHA, New York Heart Association.
\end{tabular}

sutureless prosthesis was implanted in 3 patients. New proper positioning was sufficient in the remaining patients; in 2 of them it was secured by adding another stitch to secure the positioning in the annular level. Retrospectively, the wrong size was causing valves to migrate into the left ventricle or ascending aorta after termination of cardiac arrest. In 2 patients with pure aortic insufficiency, the sutureless prosthesis slid into the left ventricle after restoring contractions and filling it with blood with consecutive extension of the elastic annulus. They were exchanged with a larger size of the sutureless valve prosthesis. In 1 patient, the prosthesis was placed too high and slid into the ascending aorta during manipulation of the heart for bleeding control. It was repositioned and secured with 2 guiding stitches tied down.

TABLE 2. Risk of mortality and outcome

\begin{tabular}{lc}
\hline Isolated AVR & \\
STS score & $12.1 \%$ (range, $1.6 \%-34.9 \% ; \mathrm{SD}, 5.8 \%)$ \\
Logistic euroSCORE & $16.3 \%$ (range, $2.1 \%-69 \% ; \mathrm{SD}, 12.6 \%)$ \\
30-d mortality & $1 / 71(1.4 \%)$ \\
Overall mortality & $2 / 71(2.8 \%)$ \\
Concomitant surgery & \\
STS score & $19 \%$ (range, $6.5 \%-75.9 \% ; \mathrm{SD}, 13.2 \%)$ \\
Logistic euroSCORE & $27.3 \%($ range, $2.9 \%-90.3 \% ; \mathrm{SD}, 24.2 \%)$ \\
30-d mortality & $7 / 49(14.3 \%)$ \\
Overall mortality & $9 / 49(18.4 \%)$ \\
\hline
\end{tabular}

AVR, Aortic valve replacement; STS, Society of Thoracic Surgeons; euroSCORE, European System for Cardiac Operative Risk Evaluation; $S D$, standard deviation.
TABLE 3. Surgical procedures

\begin{tabular}{lr}
\hline Overall Enable (Medtronic, Inc, & 120 \\
Minneapolis, Minn) implantations & \\
Isolated AVR & $\mathbf{7 1}$ \\
- Redo AVR & 2 \\
- Redo after CABG & 2 \\
Concomitant cardiovascular procedures & 49 \\
- AVR + CABG & 30 \\
- AVR + atrial ablation & 9 \\
- AVR + ASD closure & 2 \\
- AVR + myotomy/myectomy & 1 \\
- AVR + MVR & 3 \\
- AVR + TVR & 1 \\
- AVR + pericardectomy & 1 \\
- AVR + ascending/arch aortic surgery & 2 \\
\hline$A V R$, Aortic valve replacement; $C A B G$, coronary artery bypass grafting; $A S D$, atrial \\
septal defect; $M V R$, mitral valve repair; $T V R$, tricuspid valve repair.
\end{tabular}

The accumulated follow-up time was 93.5 patient-years in total. The mean follow-up time was 313 days until March 2012. The mean hospital stay was 9 days (range, 3-89 days). Follow-up was complete in $95 \%$ of patients.

The overall 30-day mortality rate was $6.7 \%(8 / 120$ patients; Table 4). There was 1 intraoperative death due to fatal tearing of the thoracic aorta during intraaortic balloon insertion in an patient with coronary artery disease and preoperative low output syndrome. One patient in the isolated AVR group died in the early postoperative period after unsuccessful resuscitation $(1.4 \%)$. In the group with

TABLE 4. Clinical outcome and adverse effects

\begin{tabular}{lc}
\hline Mortality & \\
Early & $8 / 120(6.7 \%)$ \\
Late & $3 / 112(2.7 \%)$ \\
Repeat aortic crossclamp & $10(8.3 \%)$ \\
- Prosthesis migration & 3 \\
- Paravalvular leakage & 7 \\
Rethoracotomy & $4(3.3 \%)$ \\
MI & 0 \\
Thromboembolism* & $1(0.8 \%)$ \\
Stroke & $1(0.8 \%)$ \\
Pneumonia & $4(3.3 \%)$ \\
Renal failure & $3(2.5 \%)$ \\
Permanent pacemaker & $8(6.7 \%)$ \\
Prosthetic endocarditis & $2(1.7 \%)$ \\
Structural valve deterioration & 0 \\
Major pVL & $2(1.7 \%)$ \\
Iatrogenic valve dislocation & $1(0.8 \%)$ \\
Minor pVL & $4(3.3 \%)$ \\
Reoperation & $5(4.2 \%)$ \\
Mean length of hospital stay & $7.9 \mathrm{~d}$ \\
\hline Late mortality = after hospital discharge, rethoracotomy due to significant bleeding. \\
Minor pvL = aortic insufficiency 0 to 1 degrees or trivial regurgitation. Major \\
pvL = aortic regurgitation greater than grade I. MI, Myocardial infarction; $p V L$, \\
paravalvular leakage. Early mortality = hospital or 30-day mortality. $*$ This patient \\
had heparin-induced thrombocytopenia postoperatively, and disseminated emboli \\
developed in both feet.
\end{tabular}


concomitant surgeries, the 30-day mortality was significantly higher $(14.3 \%)$, expressing the clinical outcome of the preoperative risk assessment (Table 2).

In the late postoperative period, 3 patients died. Of those, 2 high-risk patients ( 1 of the isolated AVR group) developed a complex postoperative course leading to intractable septic multiorgan failure (days 31 and 48). Another patient with concomitant coronary bypass grafting died at home of an unknown reason after 82 days. In those patients who died during the observation period, the corresponding logistic European System for Cardiac Operative Risk Evaluation and Society of Thoracic Surgeons scores were a mean $51.4 \%$ and $15.3 \%$ for isolated AVR and $45.6 \%$ and $40 \%$ for concomitant cardiac surgeries, respectively.

As shown in Table 4, the reoperation rate was 4.2\% (5/ 120 patients). Replacement of the Enable prosthesis took place on days 5, 21, 35, 52, and 105 after the implantation procedure. The earliest reoperation took place in a patient in whom manipulation at the aortic root had to be performed to control bleeding from the aortotomy site during a rethoracotomy. This caused an active extraction of the prosthesis from its annular position because of interference with the nitinol stent by placement of a suture accidentally through the nitinol stent, leading to upward traction. After repeat aortic crossclamp to reposition the Enable prosthesis correctly, a major paravalvular leakage occurred in 1 patient during the postoperative course, requiring a reoperation. Another patient had prosthetic endocarditis and systemic septic impairment. In 2 other patients, the underlying aortic valve pathology revealed a pure aortic insufficiency. A major paravalvular leakage was discovered by echocardiography examination soon after the operation and deteriorated further. In all reoperated patients, an uncomplicated exchange with a nonsutureless type of prosthesis was performed with no mortality. The explanted Enable valve was carefully examined but did not show any structural defect or thrombotic formations.

Permanent pacemaker therapy after Enable implantation was necessary in 8 patients postoperatively $(6.7 \%)$ because of newly developed arrhythmia. There was no difference between patients with isolated AVR or concomitant cardiac procedures ( $7 \%$ vs $6.1 \%$ ).

At the time of discharge, transthoracic echocardiogram revealed peak pressure gradients of $14.9 \pm 4.5 \mathrm{~mm} \mathrm{Hg}$, mean pressure gradients of $8.6 \pm 2.8 \mathrm{~mm} \mathrm{Hg}$, and aortic valve area of mean $2.5 \pm 0.9 \mathrm{~cm}^{2}$. During 6- and 12-month follow-ups, the pressure gradients remained constant. Peak pressure was $16 \pm 5.1 \mathrm{~mm} \mathrm{Hg}$ and $16.5 \pm 5.8 \mathrm{~mm} \mathrm{Hg}$, and mean pressure was $9.3 \pm 3.6 \mathrm{~mm} \mathrm{Hg}$ and $9.5 \pm 3.8 \mathrm{~mm} \mathrm{Hg}$ at 6 and 12 months, respectively (Figure 1). Mean aortic valve area had decreased at the same time period to $2.2 \pm 0.8 \mathrm{~cm}^{2}$.

Examination by echocardiography at the time of discharge detected 4 trivial paravalvular leakages, which had not appeared intraoperatively and remained stable during

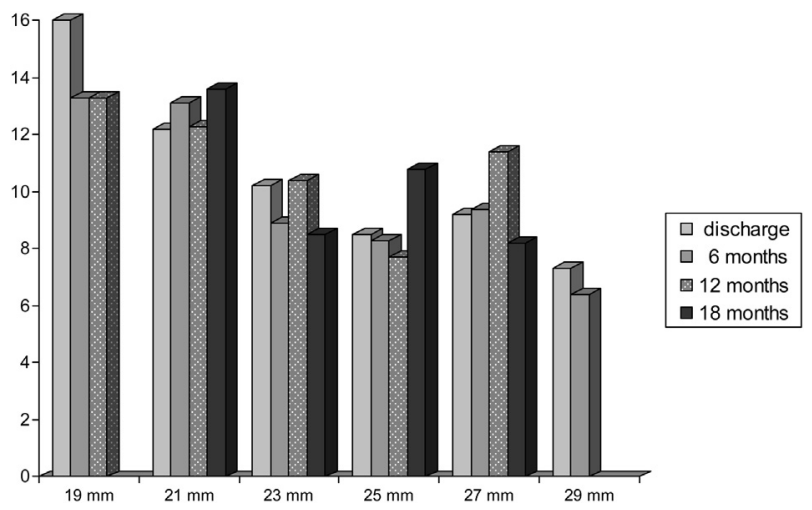

FIGURE 1. Mean pressure gradient according to various prosthesis sizes during follow-up.

follow-up without any clinical impairment and signs of hemolysis.

\section{DISCUSSION}

Adequate treatment of patients with severe aortic valve disease remains challenging for both interventional and surgical approaches. Despite the progressive development of transcatheter aortic valve interventions, the midterm data are not convincing. The PARTNER trial (cohorts A and B) has shown some benefit in patients assigned as inoperable, but medical, ethical, and financial concerns are raised. ${ }^{13,14}$ It is a constant methodical risk for mortality and morbidity that the usually heavy calcified leaflets and annulus are pushed aside, causing a high incidence of embolic events. Furthermore, the results remain questionable in many cases; calcifications still in place are causing an incomplete attachment to the annulus and, consecutively, paravalvular leakages of higher degree in up to $70 \%$ of cases. ${ }^{15,16}$

The sutureless implantation technique represents a novel development in innovative surgical AVR. The possibility of avoiding multiple stitches at the (potentially calcified) aortic annulus ensures less traumatic surgery at the aortic root, eventually causing less embolic complications. In fact, embolic complications in this study were rare. Insufficient peripheral vascular perfusion due to heparin-induced thrombocytopenia developed in only 1 patient with postoperative septic complications. Our anticoagulation protocol after sutureless valve implantation consists of clopidogrel for 6 weeks and long-term low-dose aspirin based on recommendations for patients with carotid artery stenting. ${ }^{17}$ Administration of a vitamin $\mathrm{K}$ antagonist is avoided if no other indications apply.

The absence of suturing offers the potential for reducing aortic crossclamp and total cardiopulmonary bypass time. We were able to slightly decrease our implantation times, as did Aymard and colleagues. ${ }^{10}$ In a multicenter study, Martens and colleagues ${ }^{9}$ reported on mean crossclamp times of $58 \pm 25$ minutes, although it is unclear whether 
those numbers are related to stand-alone AVR only. In an ideal situation, aortic crossclamp times less than 20 minutes are feasible.

In our experience, sutureless aortic valve implantation offers potential advantages in high-risk patients or for those with concomitant procedures. The overall mean aortic crossclamp time in our group was $47 \pm 19$ minutes, considering the fact that $41 \%$ underwent additional partly extensive cardiac procedures (Table 2).

On the other hand, correct positioning of the prosthesis can be time-consuming and must be carried out accurately. The question of whether any paravalvular leakage, observed by intraoperative TEE, must be corrected or not, is controversial. It is doubtless that a clearly visible paravalvular leakage at the time of surgery demands immediate correction.

In our series, the incidence of repeat crossclamp of the aorta to reposition the sutureless prosthesis because of immediate paravalvular leakage was $8.3 \%$. This represents a unique feature in this novel surgical approach for AVR. It confirms the necessity of precise sizing, which is difficult in specific constellations, such as pure aortic insufficiency and irregular length and level of the sinuses. In critical cases, single stay stitches may be used to avoid migration from the aortic annulus and consecutive paravalvular leakage. In our experience, repeat crossclamp to correct prosthesis misplacement did not result in a worse outcome or extensive prolongation of operating times. Therefore, it is our policy that no patient leaves the operating room without a perfect echocardiographic result, that is, there is no acceptance of any paravalvular leak.

We have observed a favorable outcome in patients who underwent isolated AVR with an overall mortality of $2.8 \%$ during the observation period of up to 20 months, although the predictive mortality has been significantly higher. In total, 11 patients $(9.2 \%)$ died in our study, mainly in the high-risk group with concomitant cardiac procedures. In the European multicenter study that included $30 \%$ of nonisolated AVR cases, the overall mortality was $12.9 \%$. Unfortunately, predictive risk scores and detailed information on both patient groups have not been provided. ${ }^{9}$ Aymard and colleagues ${ }^{10}$ reported on a 30-day mortality of $3.5 \%$ in 28 patients with stand-alone sutureless AVR but observed an overall 1-year mortality of $13.8 \%$. Because the Enable bioprosthesis has been proven to show persistent excellent hemodynamic results (Figure 1), other non-valverelated factors (eg, age and comorbidities) account for higher mortality rates during long-term follow-up.

In an extensive analysis, Matthews and colleagues ${ }^{18}$ reported on an incidence of postoperative pacemaker implantation after conventional AVR between 3.0\% and 11.8\%. Actual single-center experience is approximately $8.2 \%$ according to Dawkins and colleagues. ${ }^{19}$ Aymard and colleagues ${ }^{10}$ reported on the requirement of pacemaker therapy in patients after sutureless AVR in more than $18 \%$, whereas
Martens and colleagues ${ }^{20}$ have reported on only 1 pacemaker implantation in their patient group with partial sternotomy access $(4.5 \%)$. In our study, only 1 of 24 patients with a less-invasive approach $(4.2 \%)$ received postoperative pacemaker therapy; the overall incidence was $6.7 \%$. We could not ascertain a logical reason for the higher pacemaker incidence compared with those who underwent concomitant procedures other than the radial forces of the nitinol stent. Technically, decalcification of the annulus did not differ between the patients.

In general, access to the aortic valve via partial sternotomy or right anterior minithoracotomy has evolved in recent years. ${ }^{21,22}$ The sutureless valve implantation represents a further step toward true minimally invasive cardiac surgery. In principle, the folded bioprosthesis mounted onto a forceps or a delivery device in general is implantable through limited access, as others have already shown in smaller patient groups. ${ }^{20,23}$ In our experience, Enable implantation via partial sternotomy results in good outcome and no surgical impairment. In contrast, access via lateral minithoracotomy may be limited by proper instrumentation, requiring further development of surgical tools.

A major advantage of the Enable prosthesis is the broad spectrum of sizes available. Especially in smaller diameters from 19 to $23 \mathrm{~mm}$, the advantages of the superior hemodynamics become evident. To avoid patient-prosthesis size mismatch, aortic root enlargement or replacement is usually necessary at clearly greater risk. ${ }^{24}$ Borger and colleagues ${ }^{25}$ reported on the superiority of stentless aortic bioprosthesis in terms of hemodynamic results and, consequently, left ventricular mass reduction, with some evidence of improved survival in comparison with patients who received stented aortic valves. In their study, the overall mean pressure gradients were mean $11 \pm 5 \mathrm{~mm} \mathrm{Hg}$ in the stentless valve group. In our experience, the low-pressure gradients even in small prosthesis sizes of $21 \mathrm{~mm}$ (compare with Figure 1) and constant mean pressure gradients between 12 and $14 \mathrm{~mm} \mathrm{Hg}$ within the first 18 months demonstrate the excellent hemodynamic profile of the Enable prosthesis. The overall mean pressure gradients were lower with 8.6 $\mathrm{mm} \mathrm{Hg}$ on average at the time of discharge, slightly increasing at 12 months follow-up to $9.5 \mathrm{~mm} \mathrm{Hg}$.

From a surgical standpoint, the higher level of aortotomy with respect to the nitinol stent may lead to certain discomfort initially. Otherwise, there has not been a published case with impaired coronary blood flow due to ostia obstruction. This can be attributed to the more meticulous positioning at the aortic annular level but is also due to design of the prosthesis itself: Expansion of the leaflets is limited to the dimension of the nitinol frame. In context with sutureless valve therapy, the nitinol stent is not only thinner but also hardly influences the left outflow tract. Nevertheless, specific attention is necessary for exact sizing because the prosthesis is fixed by radial forces of the nitinol frame at the 
annular level only. This feature allows for use of this particular prosthesis with slight post-stenotic dilatation, too.

\section{CONCLUSIONS}

This single-center experience in sutureless aortic valve implantation represents an innovative surgical treatment for patients with severe aortic valve disease, resulting in excellent persistent hemodynamic conditions. The implantation procedure is safe and surgically effective for AVR. We believe the spectrum of indications for use of the sutureless valve to be broad, but certainly more experience with long-term follow-up will be necessary to verify the durability and safety of the valve prosthesis. We see advantages of the sutureless approach in underlying patient-prosthesis size mismatch, severely calcified aortic roots in high-risk patients, and potentially in true minimally invasive surgeries. The procedure can be time-saving and advantageous in extensive surgery and high-risk patients.

\section{References}

1. Pibarot P. Valvular disease in 2010. Evolution and revolution in risk stratification and therapy. Nat Rev Cardiol. 2011;8:76-8.

2. Jamieson WR, Riess FC, Raudkivi PJ, Metras J, Busse EF, Goldstein J, et al. Medtronic Mosaic porcine bioprosthesis: assessment of 12-year performance. J Thorac Cardiovasc Surg. 2011;142:302-7.

3. Chan V, Kulik A, Tran A, Hendry P, Masters R, Mesana TG, et al. Long-term clinical and hemodynamic performance of the Hancock II versus the Perimount aortic bioprostheses. Circulation. 2010;122:S10-6.

4. Aicher D, Langer F, Lausberg H, Bierbach B, Schäfers HJ. Aortic root remodeling: ten-year experience with 274 patients. J Thorac Cardiovasc Surg. 2007;134:909-15.

5. Dapunt OE, Easo J, Hölzl PP, Murin P, Südkamp M, Horst M, et al. Stentless full root bioprosthesis in surgery for complex aortic valve-ascending aortic disease: a single center experience of over 300 patients. Eur J Cardiothorac Surg. 2008;33:554-9.

6. David TE, Feindel CM, Webb GD, Colman JM, Armstrong S, Maganti M. Longterm results of aortic valve-sparing operations for aortic root aneurysm. J Thorac Cardiovasc Surg. 2006;132:347-54.

7. Levy D, Garrison RJ, Savage DD, Kannel WB, Castelli WP. Left ventricular mass and incidence of coronary heart disease in an elderly cohort: the Framingham Heart Study. Ann Intern Med. 1989;110:101-7.

8. De Paulis R, Sommariva L, Colagrande L, De Matteis GM, Fratini S, Tomai F, et al. Regression of left ventricular hypertrophy after aortic valve replacement for aortic stenosis with different valve substitutes. J Thorac Cardiovasc Surg. 1998;116:590-8.
9. Martens S, Sadowski J, Eckstein FS, Bartus K, Kapelak B, Sievers HH, et al. Clinical experience with the ATS $3 \mathrm{f}$ Enable sutureless bioprosthesis. Eur J Cardiothorac Surg. 2011;40:749-55.

10. Aymard T, Kadner A, Walpoth N, Gober V, Englberger L, Stalder M, et al. Clinical experience with the second-generation 3 f Enable sutureless aortic valve prosthesis. J Thorac Cardiovasc Surg. 2010;140:313-6.

11. Martens S, Ploss A, Sirat S, Miskovic A, Moritz A, Doss M. Sutureless aortic valve replacement with the 3 f Enable Aortic Bioprosthesis. Ann Thorac Surg. 2009;87:1914-8

12. Sadowski J, Kapelak B, Pfitzner R, Bartus K. Sutureless aortic valve bioprosthesis '3f/ATS Enable' - 4.5 years of single centre experience. Kardiol Pol. 2009;67:956-63.

13. Leon MB, Smith CR, Mack M, Mill DC, Moses JW, Svensson LG, et al. Transcatheter aortic-valve implantation for aortic stenosis in patients who cannot undergo surgery. N Engl J Med. 2010;363:1597-607.

14. Smith C, Leon M, Mack M, Miller D, Moses J, Svensson L, et al. PARTNER Trial Investigators. Transcatheter versus surgical aortic-valve replacement in high-risk patients. N Engl J Med. 2011;364:2187-98.

15. Sherif MA, Abdel-Wahab M, Stocker B, Geist V, Richardt D, Tolg R, et al. Anatomic and procedural predictors of paravalvular aortic regurgitation after implantation of the Medtronic CoreValve bioprosthesis. J Am Coll Cardiol. 2010; 56:1623-9.

16. Webb JG, Altwegg L, Boone RH, Cheung A, Ye J, Lichtenstein S, et al. Transcatheter aortic valve implantation: impact on clinical and valve-related outcomes. Circulation. 2009;119:3009-16.

17. Diener HC, Bogousslavsky J, Brass LM, Cimminiello C, Csiba L, Kaste M, et al. Aspirin and clopidogrel compared with clopidogrel alone after recent ischaemic stroke or transient ischaemic attack in high-risk patients (MATCH): randomised double- blind, placebo-controlled trial. Lancet. 2004;364:331-7.

18. Matthews IG, Fazal IA, Bates MG, Turley AJ. In patients undergoing aortic valve replacement, what factors predict the requirement for permanent pacemaker implantation? Interact Cardiovasc Thorac Surg. 2011;12:475-9.

19. Dawkins S, Hobson AR, Kalra PR, Tang AT, Monro JL, Dawkins KD. Permanen pacemaker implantation after isolated aortic valve replacement: incidence, indications, and predictors. Ann Thorac Surg. 2008;85:108-12.

20. Martens S, Zierer A, Ploss A, Sirat S, Miskovic A, Moritz A, et al. Sutureless aortic valve replacement via partial sternotomy. Innovations. 2010;5:12-5

21. Soltesz EG, Cohn LH. Minimally invasive valve surgery. Cardiol Rev. 2007;15 109-15.

22. Brown ML, McKellar SH, Sundt TM, Schaff HV. Ministernotomy versus conventional sternotomy for aortic valve replacement: a systematic review and metaanalysis. J Thorac Cardiovasc Surg. 2009;137:670-9.

23. Glauber M, Miceli A, Bevilacqua S, Farneti PA. Minimally invasive aortic valve replacement via right anterior minithoracotomy: early outcomes and midterm follow-up. J Thorac Cardiovasc Surg. 2011;142:1577-9.

24. Sommers KE, David TE. Aortic valve replacement with patch enlargement of the aortic annulus. Ann Thorac Surg. 1997;63:1608-12.

25. Borger MA, Carson SM, Ivanov J, Rao V, Scully HE, Feindel CM, et al. Stentless aortic valves are hemodynamically superior to stented valves during mid-term follow-up: a large retrospective study. Ann Thorac Surg. 2005;80:2180-5. 